\title{
Analysis of Multipath and CW Interference Effects on GNSS Receivers with EMLP Discriminator
}

\author{
Bo Qu ${ }^{1}$, Jiaolong $\mathrm{Wei}^{1}$, Shuangna Zhang ${ }^{2}$, Liang $\mathrm{Bi}^{2}$ \\ ${ }^{1}$ Department of Electronic and Information Engineering, Huazhong University of Science and Technology, China \\ ${ }^{2}$ Space Star Technology Co. Ltd, China \\ Email: qubo@hust.edu.cn, jlwei@mail.hust.edu.cn
}

Received May, 2013

\begin{abstract}
Multipath and continuous wave (CW) interference may cause severe performance degradation of global navigation satellite system (GNSS) receivers. This paper analyzes the code tracking performance of early-minus-late power (EMLP) discriminator of GNSS receivers in the presence of multipath and CW interference. An analytical expression of the code tracking error is suggested for EMLP discriminator, and it can be used to assess the effect of multipath and CW interference. The derived expression shows that the combined effects include three components: multipath component; CW interference component and the combined component of multipath and CW interference. The effect of these components depends on some factors which can be classified into two categories: the receiving environment and the receiver parameters. Numerical results show how these factors affect the tracking performances. It is shown that the proper receiver parameters can suppress the combined effects of multipath and CW interference.
\end{abstract}

Keywords: Multipath; Interference; GNSS; EMLP; Discriminator

\section{Introduction}

GNSS signal is very susceptibility to receiving environment [1-2]. Multipath is the dominant error source in most GNSS applications. Therefore, multipath performance analysis plays a significant role in the analysis of the code tracking performance of GNSS receivers. The multipath error envelope (MEE) is a common way of assessing the multipath performance of a given sig$\mathrm{nal} /$ receiver combination [3]. CW interference is typical radio frequency interference (RFI) which is another error source for GNSS receivers [4, 5]. The post-correlati on effects of narrowband interference and partial-band interference have been analyzed in [4, 6]. Reference [7] suggested analytical expressions for GNSS receiver performance such as the effective carrier-to-noise density ratio, the code tracking error and the carrier phase tracking error for the receiver affected by CW interference. Reference [8] presented the analytic expressions of the code tracking error bound for the EMLP discriminator and the dot-product (DP) discriminator. The definition of new families of curves named interference error envelope (IEE) and interference running average (IRA) was presented, and these tools are able to assess the impact of RF interference on different GNSS receivers [9].

The effects of multipath or interference have been analyzed in the articles above. However, if multipath and interference exist at the same time, their effects on the code tracking performance with EMLP discriminator are not independent. However, the combined effects of multipath and CW interference haven't been analyzed in other papers. This paper analyzes the effects of multipath and CW interference on the code tracking performance of GNSS receivers. An analytical expression of the code tracking error is suggested for EMLP discriminator, and it can be used to assess the combined effect of multipath and CW interference.

The rest of this paper is organized as follows. Signal models are provided in Section 2. The analytical expression of the code tracking error for EMLP discriminator is derived in Section 3. Section 4 shows how the receiving environment and the receiver parameters affect the code tracking error. This paper concludes in Section 5.

\section{Signal Models}

As defined in [10], the direct signal is denoted as equation(1):

$$
r_{0}(t)=a_{0} s\left(t-\tau_{0}\right) \cos \left(2 \pi f_{c} t+\varphi_{0}\right)
$$

with $a_{0}$ being the amplitude of the direct signal, $s(t)$ being the pseudo random noise (PRN) code, $\tau_{0}$ being the time delay, $f_{c}$ being the carrier frequency, and $\varphi_{0}$ being the carrier phase. 
With the increase in the number of reflections, the amplitudes of reflected signals are becoming smaller and smaller. Without loss of generality, one reflected signal with the maximum amplitude is taken into consideration to simplify the received signal model, so the reflected signal can be simplified as follow:

$$
r_{N}(t)=a_{1} s\left(t-\tau_{1}\right) \cos \left(2 \pi f_{c} t+\varphi_{1}\right)
$$

where $a_{1}$ is the amplitude of a reflected signal, $\tau_{1}$ is the time delay of a reflected signal, $\varphi_{1}$ is the carrier phase of a reflected signal.

The CW interference can be considered to be a sine wave which can be expressed as:

$$
l(t)=c_{l} \cos \left(2 \pi f_{c} t+2 \pi f_{l} t+\varphi_{l}\right)
$$

where $c_{l}$ is the amplitude of CW interference, $f_{l}$ is the frequency offset from the carrier frequency $f_{c}$, and $\varphi_{l}$ is the phase of CW interference.

Therefore, the received signal can be expressed as:

$$
\begin{aligned}
r(t)= & a_{0} s\left(t-\tau_{0}\right) \cos \left(2 \pi f_{c} t+\varphi_{0}\right) \\
& +a_{1} s\left(t-\tau_{1}\right) \cos \left(2 \pi f_{c} t+\varphi_{1}\right) \\
& +c_{l} \cos \left(2 \pi f_{c} t+2 \pi f_{l} t+\varphi_{l}\right)
\end{aligned}
$$

Before processing the received signal and beginning the code tracking, the received signal needs to be downconverted. In the down converting process, the estimated carrier phase is provided by the phase locked loop (PLL) and a replica carrier is generated by the receiver. The replica carrier is expressed as $2 \cos \left(2 \pi f_{c} t+\hat{\varphi}_{0}\right)$, where $\hat{\varphi}_{0}$ is the estimated carrier phase. After down converting and filtering the received signal, the received signal can be expressed by the in-phase component and the quadrature component. The in-phase component can be expressed as follow:

$$
\begin{aligned}
s_{I}(t)= & a_{0} s\left(t-\tau_{0}\right) \cos \left(\varphi_{0}-\hat{\varphi}_{0}\right) \\
& +a_{1} s\left(t-\tau_{1}\right) \cos \left(\varphi_{1}-\hat{\varphi}_{0}\right)+c_{l} \cos \left(2 \pi f_{l} t+\varphi_{l}-\hat{\varphi}_{0}\right)
\end{aligned}
$$

The quadrature component can be expressed as follow:

$$
\begin{aligned}
s_{Q}(t) & =a_{0} s\left(t-\tau_{0}\right) \sin \left(\varphi_{0}-\hat{\varphi}_{0}\right) \\
& +a_{1} s\left(t-\tau_{1}\right) \sin \left(\varphi_{1}-\hat{\varphi}_{0}\right)+c_{l} \sin \left(2 \pi f_{l} t+\varphi_{l}-\hat{\varphi}_{0}\right)
\end{aligned}
$$

In the delay lock loop, the code generator usually generates two types of PRN codes: early replica code $s^{*}\left(t-\hat{\tau}_{0}+d / 2\right)$, late replica code $s^{*}\left(t-\hat{\tau}_{0}-d / 2\right)$, where $\hat{\tau}_{0}$ is the estimated time delay, * denotes the conjugate operation, and $d$ is the correlator spacing. In the code tracking process, the replica codes are multiplied and integrated with the in-phase component and the quadrature component.

The early in-phase output $I_{E}$ can be expressed as follow:

$$
\begin{aligned}
I_{E}= & a_{0} \cos \left(\hat{\varphi}_{0}-\varphi_{0}\right) R\left(\varepsilon_{\tau}-\frac{d}{2}\right) \\
& +a_{1} \cos \left(\varphi_{1}-\hat{\varphi}_{0}\right) R\left(\varepsilon_{\tau}-\hat{\tau}_{1}-\frac{d}{2}\right) \\
& +\frac{c_{l}}{T_{p}} \int_{-T_{p} / 2}^{T_{p} / 2} \cos \left(2 \pi f_{l} t+\varphi_{l}-\hat{\varphi}_{0}\right) s_{I}^{*}\left(t-\hat{\tau}_{0}+\frac{d}{2}\right) d t
\end{aligned}
$$

Similarly, the late in-phase output $I_{L}$, the early quadrature output $Q_{E}$ and the late quadrature output $Q_{L}$ can be expressed respectively as follow:

$$
\begin{aligned}
I_{L}= & a_{0} \cos \left(\hat{\varphi}_{0}-\varphi_{0}\right) R\left(\varepsilon_{\tau}+\frac{d}{2}\right) \\
& +a_{1} \cos \left(\varphi_{1}-\hat{\varphi}_{0}\right) R\left(\varepsilon_{\tau}-\hat{\tau}_{1}+\frac{d}{2}\right) \\
& +\frac{c_{l}}{T_{p}} \int_{-T_{p} / 2}^{T_{p} / 2} \cos \left(2 \pi f_{l} t+\varphi_{l}-\hat{\varphi}_{0}\right) s^{*}\left(t-\hat{\tau}_{0}-\frac{d}{2}\right) d t \\
Q_{E}= & a_{0} \sin \left(\hat{\varphi}_{0}-\varphi_{0}\right) R\left(\varepsilon_{\tau}-\frac{d}{2}\right) \\
& +a_{1} \sin \left(\hat{\varphi}_{0}-\varphi_{1}\right) R\left(\varepsilon_{\tau}-\hat{\tau}_{1}-\frac{d}{2}\right) \\
& -\frac{c_{l}}{T_{p}} \int_{-T_{p} / 2}^{T_{p} / 2} \sin \left(2 \pi f_{l} t+\varphi_{l}-\hat{\varphi}_{0}\right) s^{*}\left(t-\hat{\tau}_{0}+\frac{d}{2}\right) d t \\
Q_{L} & a_{0} \sin \left(\hat{\varphi}_{0}-\varphi_{0}\right) R\left(\varepsilon_{\tau}+\frac{d}{2}\right) \\
& +a_{1} \sin \left(\hat{\varphi}_{0}-\varphi_{1}\right) R\left(\varepsilon_{\tau}-\hat{\tau}_{1}+\frac{d}{2}\right) \\
& -\frac{c_{l}}{T_{p}} \int_{-T_{p} / 2}^{T_{p} / 2} \sin \left(2 \pi f_{l} t+\varphi_{l}-\hat{\varphi}_{0}\right) s^{*}\left(t-\hat{\tau}_{0}-\frac{d}{2}\right) d t
\end{aligned}
$$

where $T_{p}$ is the integration time, $\varepsilon_{\tau}=\hat{\tau}_{0}-\tau_{0}$ is the code tracking error, and $\hat{\tau}_{1}=\tau_{1}-\tau_{0}$ is the extra delay of the reflected signal with respect to the direct signal, $R(\cdot)$ is the autocorrelation function defined as follow:

$$
R(\tau)=\frac{1}{T_{p}} \int_{-T_{p} / 2}^{T_{p} / 2} s(t) s^{*}(t-\tau) d t
$$

When CW interference is multiplied with the replica PRN signal, the CW interference is spread by the PRN code. Since the code tracking loop is an equivalent lowpass filter, when the interference locates at zero frequency (this occurs when $f_{l}=n /\left(N_{c} T_{c}\right)$ ), it causes the most serious degradation of the code tracking performance. Then the interference term of $I_{E}$ can be expressed as follow [8]:

$$
I_{E_{-} l}=c_{l}|C(n)| \cos \left(\alpha+\pi d n / N_{c} T_{c}\right)
$$

where $C(n)=S_{p}(n) \sum_{m=0}^{N_{c}-1} c_{m} e^{-j 2 \pi m n / N_{c}} / N_{c} T_{c}$ 
is the Fourier transform of the PRN signal, $S_{p}(n)$ is the transform of the pluse shape,

$$
\alpha=\varphi_{l}-\hat{\varphi}_{0}+\beta+2 \pi\left(n / N_{c} T_{c}\right) \hat{\tau}_{0},
$$

and $\beta$ is the phase of $C(-n)$. Similarly, we can obtain the interference term of $I_{L}, Q_{E}$, and $Q_{L}$.

\section{Code Tracking Performance Analysis}

The EMLP discriminator is a typical noncoherent discriminator which can mitigate the effect of the phase difference between the carrier phase and the estimated carrier phase. The output of EMLP discriminator is denoted as follow:

$$
D_{E M L P}=\left(I_{E}^{2}+Q_{E}^{2}\right)-\left(I_{L}^{2}+Q_{L}^{2}\right)
$$

Assuming that the carrier tracking loop tracks the carrier phase of the received signal perfectly, we can obtain $\varphi_{0}=\hat{\varphi}_{0}$. Substituting (7)-(10) into (13) and simplifying the expression, we can obtain the following expression.

$$
\begin{aligned}
D_{E M L P}\left(\varepsilon_{\tau}\right) & =D_{\text {Direct }}\left(\varepsilon_{\tau}\right)+D_{\text {Mult }}\left(\varepsilon_{\tau}\right) \\
& +D_{\text {Infer }}\left(\varepsilon_{\tau}\right)+D_{\text {Mult_Infer }}\left(\varepsilon_{\tau}\right)
\end{aligned}
$$

The output of EMLP discriminator includes four components: the direct signal component $D_{\text {Direct }}\left(\varepsilon_{\tau}\right)$, the multipath component $D_{\text {Mult }}\left(\varepsilon_{\tau}\right)$, the $\mathrm{CW}$ interference component $D_{\text {Infer }}\left(\varepsilon_{\tau}\right)$, and the combined component $D_{\text {Mult_Infer }}\left(\varepsilon_{\tau}\right)$ of multipath and CW interference.

The direct signal component $D_{\text {Direct }}\left(\varepsilon_{\tau}\right)$ can be expressed as follow:

$$
D_{\text {Direct }}\left(\varepsilon_{\tau}\right)=a_{0}^{2}\left[R^{2}\left(\varepsilon_{\tau}-\frac{d}{2}\right)-R^{2}\left(\varepsilon_{\tau}+\frac{d}{2}\right)\right]
$$

The direct signal component has relation to the amplitude of the direct signal $a_{0}$, the correlator spacing d, and the autocorrelation function of the navigation signal. The direct signal component is the output of EMLP discriminator without the effects of multipath and CW interference.

The multipath component $D_{\text {Mult }}\left(\varepsilon_{\tau}\right)$ can be expressed as follow:

$$
\begin{aligned}
D_{\text {Mult_EMLP }}\left(\varepsilon_{\tau}\right)= & a_{1}^{2}\left[R^{2}\left(\varepsilon_{\tau}-\hat{\tau}_{1}-\frac{d}{2}\right)-R^{2}\left(\varepsilon_{\tau}-\hat{\tau}_{1}+\frac{d}{2}\right)\right] \\
+2 a_{0} a_{1} \cos \left(\hat{\varphi}_{1}\right) & {\left[R\left(\varepsilon_{\tau}-\frac{d}{2}\right) R\left(\varepsilon_{\tau}-\hat{\tau}_{1}-\frac{d}{2}\right)\right.} \\
& \left.-R\left(\varepsilon_{\tau}+\frac{d}{2}\right) R\left(\varepsilon_{\tau}-\hat{\tau}_{1}+\frac{d}{2}\right)\right]
\end{aligned}
$$

In addition to the amplitude of the direct signal $a_{0}$, the correlator spacing $d$ and the autocorrelation function of the navigation signal, equation (16) shows that the multipath component $D_{\text {Mult }}\left(\varepsilon_{\tau}\right)$ has relation to the amplitude $a_{l}$ of the reflected signal, the phase difference of the reflected signal and the direct signal, and the extra delay of the reflected signal with respect to the direct signal $\hat{\tau}_{1}$. When only multipath signal exists in the receiving environment, the output of EMLP discriminator is the sum of the multipath component $D_{\text {Mult }}\left(\varepsilon_{\tau}\right)$ and the direct signal component $D_{\text {Direct }}\left(\varepsilon_{\tau}\right)$.

The CW interference component $D_{\text {Infer }}\left(\varepsilon_{\tau}\right)$ can be expressed as follow:

$$
\begin{aligned}
D_{\text {Infer }}\left(\varepsilon_{\tau}\right)= & 2 a_{0} A_{c w} \cos (\alpha) \cos \left(\pi d n / N_{c} T_{c}\right) \\
& \times\left[R\left(\varepsilon_{\tau}-\frac{d}{2}\right)-R\left(\varepsilon_{\tau}+\frac{d}{2}\right)\right] \\
& +2 a_{0} A_{c w} \sin (\alpha) \sin \left(\pi d n / N_{c} T_{c}\right) \\
& \times\left[R\left(\varepsilon_{\tau}-\frac{d}{2}\right)+R\left(\varepsilon_{\tau}+\frac{d}{2}\right)\right]
\end{aligned}
$$

CW interference component $D_{\text {Infer }}\left(\varepsilon_{\tau}\right)$ has relation to the phase $\alpha=\varphi_{l}-\hat{\varphi}_{0}+\beta+2 \pi\left(n / N_{c} T_{c}\right) \hat{\tau}_{0}$, the amplitude and the frequency of $\mathrm{CW}$ interference beside the amplitude of the direct signal $a_{0}$, the correlator spacing $\mathrm{d}$ and the autocorrelation function of the navigation signal. It is easy to know that the output of EMLP discriminator is the sum of the CW interference component $D_{\text {Infer }}\left(\varepsilon_{\tau}\right)$ and the direct signal component $D_{\text {Direct }}\left(\varepsilon_{\tau}\right)$ in the case that only $\mathrm{CW}$ interference exists in the receiving environment.

The combined component $D_{\text {Mult_Infer }}\left(\varepsilon_{\tau}\right)$ of multipath and CW interference can be expressed as equation (18).

$$
\begin{aligned}
& D_{\text {Mult_Infer_EMLP }}\left(\varepsilon_{\tau}\right) \\
= & 2 a_{1} A_{c w} \cos \left(\hat{\varphi}_{1}+\alpha\right) \cos \left(\pi d n / N_{c} T_{c}\right) \\
& \times\left[R\left(\varepsilon_{\tau}-\hat{\tau}_{1}-\frac{d}{2}\right)-R\left(\varepsilon_{\tau}-\hat{\tau}_{1}+\frac{d}{2}\right)\right] \\
& +2 a_{1} A_{c w} \sin \left(\hat{\varphi}_{1}+\alpha\right) \sin \left(\pi d n / N_{c} T_{c}\right) \\
& \times\left[R\left(\varepsilon_{\tau}-\hat{\tau}_{1}-\frac{d}{2}\right)+R\left(\varepsilon_{\tau}-\hat{\tau}_{1}+\frac{d}{2}\right)\right]
\end{aligned}
$$

The combined component $D_{\text {Mult_Infer }}\left(\varepsilon_{\tau}\right)$ has relation to not only the multipath factors but also the CW interference factors. Because of the combined component $D_{\text {Mult_Infer }}\left(\varepsilon_{\tau}\right)$, the effects of multipath and CW interference are not independent.

The tracking error is usually small, so $D_{E M L P}\left(\varepsilon_{\tau}\right)$ can be linearly expressed as follow:

$$
D_{E M L P}\left(\varepsilon_{\tau}\right) \approx D_{E M L P}(0)+\varepsilon_{\tau} D_{E M L P}^{\prime}(0)
$$

The tracking error $\varepsilon_{\tau}$ can be expressed as

$$
\varepsilon_{\tau}=-\frac{D_{E M L P}(0)}{D_{E M L P}^{\prime}(0)}
$$

In order to simplify the mathematic derivation of the tracking error, some functions are defined as equations (21)-(24). 


$$
\begin{gathered}
R A\left(\hat{\tau}_{1}, d\right)=R\left(\hat{\tau}_{1}+\frac{d}{2}\right)+R\left(\hat{\tau}_{1}-\frac{d}{2}\right) \\
R M\left(\hat{\tau}_{1}, d\right)=R\left(\hat{\tau}_{1}-\frac{d}{2}\right)-R\left(\hat{\tau}_{1}+\frac{d}{2}\right) \\
\operatorname{DRA}\left(\hat{\tau}_{1}, d\right)=R^{\prime}\left(-\hat{\tau}_{1}-\frac{d}{2}\right)+R^{\prime}\left(-\hat{\tau}_{1}+\frac{d}{2}\right)
\end{gathered}
$$

$$
\operatorname{DRM}\left(\hat{\tau}_{1}, d\right)=R^{\prime}\left(-\hat{\tau}_{1}-\frac{d}{2}\right)-R^{\prime}\left(-\hat{\tau}_{1}+\frac{d}{2}\right)
$$

where $R^{\prime}(\tau)$ is the derivative of $R(\tau)$.

Substituing equations (14)-(18) into equation (20), the analytical expression of the code tracking error can be derived, which is denoted as equation (25):

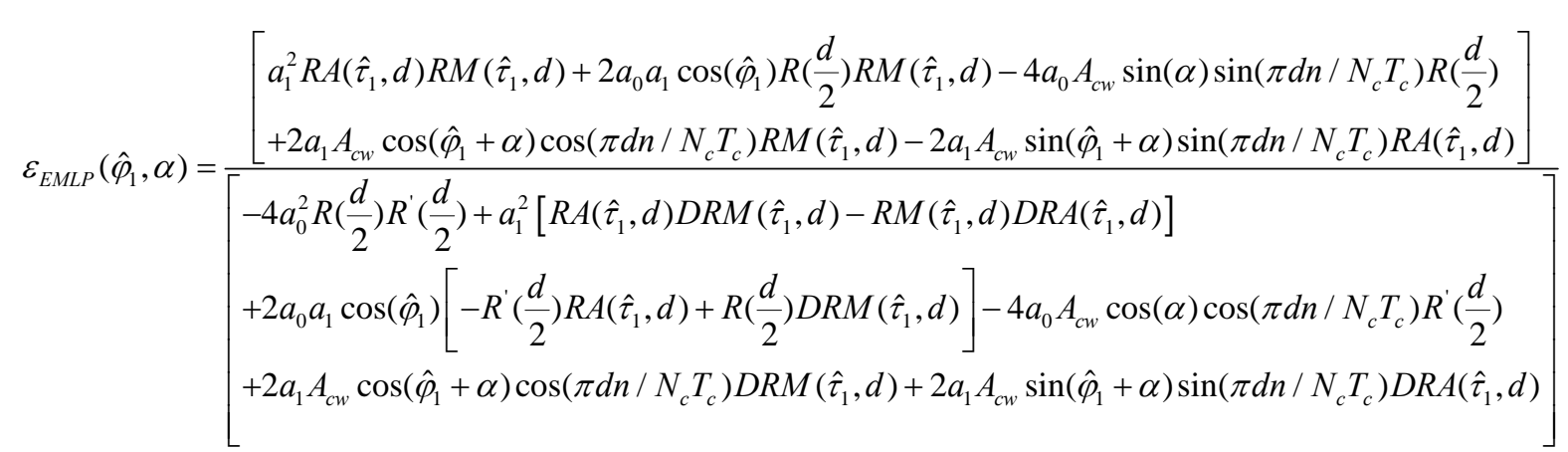

Equation (25) shows that the tracking error has realtion to the phase $\alpha$ and the phase difference $\hat{\varphi}_{1}$. The phase difference $\hat{\varphi}_{1}$ depends on the carrier phase of the reflected signal, and the phase $\alpha$ has relation to the spectrum of the PRN code signal, the phase and the frequency of the CW interference. We can make use of the maximum and the minimum values of the tracking error to evaluate the effects of the reflected signal and the CW interference. The interference and multipath error envelope (IMEE) is defined as the maximum and the minimum values of the tracking error, which can be expressed as equation (26).

$$
\varepsilon_{\text {Envelope }}=\left\{\begin{array}{l}
\operatorname{Max}\left[\varepsilon_{E M L P}\left(\hat{\varphi}_{1}, \alpha\right)\right] \\
\operatorname{Min}\left[\varepsilon_{E M L P}\left(\hat{\varphi}_{1}, \alpha\right)\right]
\end{array}\right.
$$

Because the expression of the code tracking error includes not only the term $\cos \left(\hat{\varphi}_{1}\right), \sin (\alpha)$ and $\cos (\alpha)$, but also $\cos \left(\hat{\varphi}_{1}+\alpha\right)$ and $\sin \left(\hat{\varphi}_{1}+\alpha\right)$, it is hard to obtain the analytical expression of IMEE. Fortunately, IMEE can be obtained by numerical methods for its evaluation.

\section{Numerical Results}

The IMEE expression shows that it depends on the extra delay of the reflected signal with respect to the direct signal, the frequency of $\mathrm{CW}$ interference, the correlator spacing, the amplitude ratio of the multipath signal and the direct signal (MDR), and the amplitude ratio of the direct signal and CW interference (SIR). The role of MDR and SIR is evident in the determination of the IMEE, so the effects of three other factors are analyzed in the following figures.

In the following analysis, we assumed that the received signal is the GPS L1 signal in which the PRN code is $\mathrm{C} / \mathrm{A}$ code and the front-end bandwidth is $20.46 \mathrm{MHz}$. The expression of $|C(n)|$ depends on the chip sequence and pulse shape. If the real chip sequence is taken into account, $|C(n)|$ fluctuates around the $\operatorname{sinc}(\mathrm{x})$ envelope. In order to show the IMEE clearly, the spectral lines are assumed to match the $\operatorname{sinc}(x)$ envelope exactly. The chipping rate Rc of C/A code is $1.023 \times 10^{6}$ chip/s, and the chip duration Tc is $1 / \mathrm{Rc}$.

The effects of different frequencies of CW interference on the code tracking error are shown in Figure 1. The correlator spacing is $0.1 \mathrm{Tc}$, the amplitude ratio $a_{l} / a_{0}$ is $-10 \mathrm{~dB}$, and $a_{0} / c_{l}$ is $-15 \mathrm{~dB}$. The path delays are respectively $7.5 \mathrm{~m}, 15 \mathrm{~m}, 30 \mathrm{~m}$. The effect of CW interference on the code tracking error is fluctuating and decreasing with the frequency of $\mathrm{CW}$ interference. When the frequency of $\mathrm{CW}$ interference locates at half of the $\mathrm{C} / \mathrm{A}$ code rate, the code tracking error reaches the worst value. When the frequency of $\mathrm{CW}$ interference is the integral multiple C/A code rate, the effect of CW interference on the code tracking error is negligible.

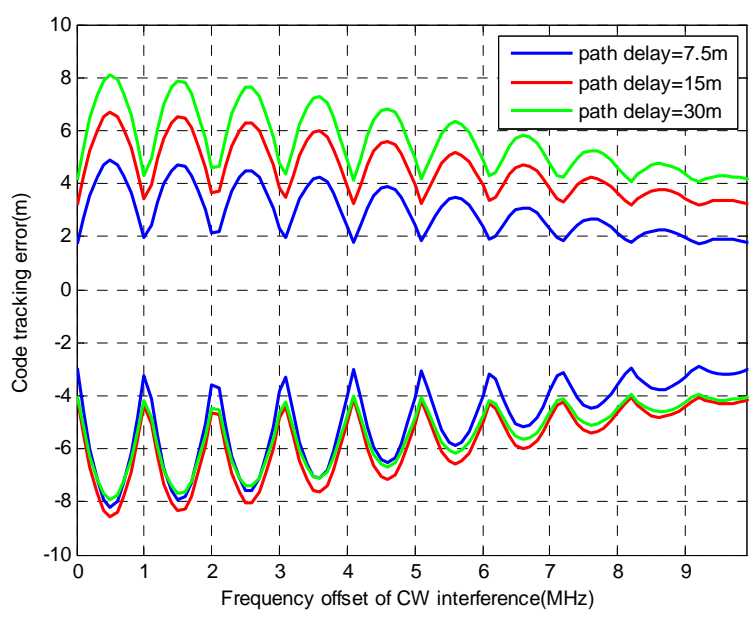

Figure 1. Code tracking error versus $f_{l}$. 
The effects of different path delays on IMEE are shown in Figure 2. The correlator spacing is $0.1 \mathrm{Tc}$, the amplitude ratio $a_{l} / a_{0}$ is $-10 \mathrm{~dB}$, and $a_{0} / c_{l}$ is $-15 \mathrm{~dB}$. The frequencies of $\mathrm{CW}$ interference are respectively $0 \mathrm{MHz}$, $0.5 \mathrm{MHz}, 1 \mathrm{MHz}, 1.5 \mathrm{MHz}$. The impact of multipath on tracking error increases, when the time delay of multipath signal increases from $0 \mathrm{~m}$. However, when the IMEE reaches the corresponding value, around which IMEE fluctuates slightly. Since the chipping duration of $\mathrm{C} / \mathrm{A}$ code is $1 /$ Rc and the path delay of 1 Tc is approximately $293 \mathrm{~m}$. When the time delay of multipath is larger than $293 \mathrm{~m}$, the multipath effect is suppressed due to the autocorrelation of C/A code.

The effects of the correlator spacing on the code tracking error are shown in Figure 3 and Figure 4. The amplitude ratio $a_{l} / a_{0}$ is $-10 \mathrm{~dB}, a_{0} / c_{l}$ is $-15 \mathrm{~dB}$, and the correlator spacing are respectively $0.1 \mathrm{Tc}, 0.3 \mathrm{Tc}$, and 0.5 Tc.

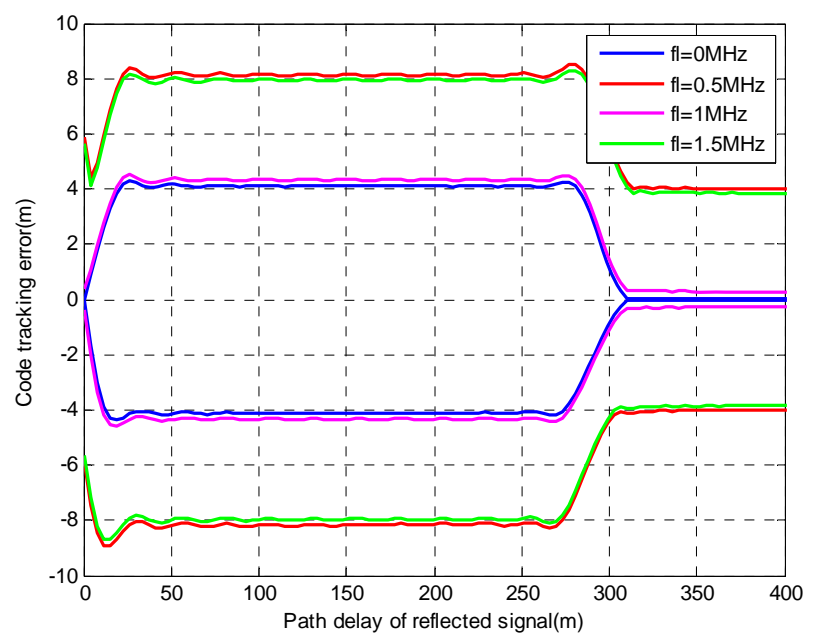

Figure 2. Code tracking error versus the path delay.

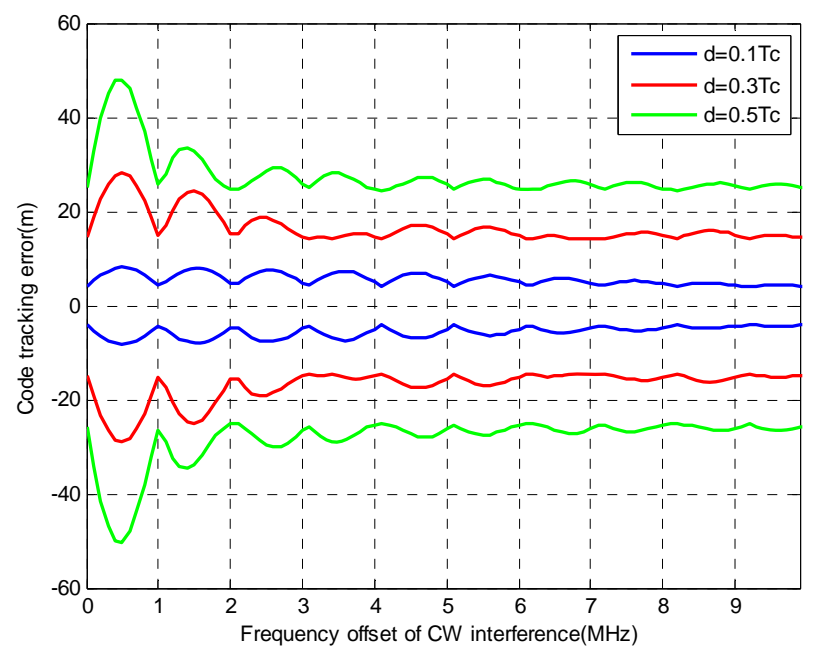

Figure 3. Code tracking error versus $f_{l}$ with different correlator spacings.

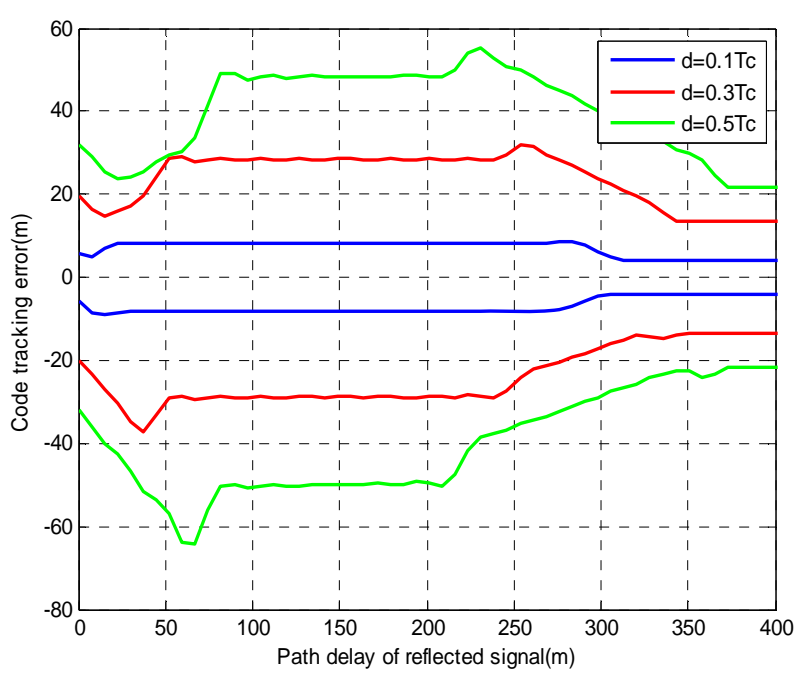

Figure 4. Code tracking error versus the path delay of the reflected signal with different correlator spacings.

\section{Conclusions}

The effects of CW interference and multipath on EMLP discriminator are analyzed in this paper. In the analysis, an analytical expression of the code tracking error is suggested for the EMLP discriminator, and it can be used to assess the combined effect of multipath and CW interference. The analytical expression of the code tracking error shows that the code tracking performance can be improved by shortening the correlator spacing for the receiver. Further, the analytical expression shows that the combined effects of CW interference and multipath on code tracking performance depend on many factors. When the frequency of CW interference locates at integral times of PRN code rate, the CW interference can be suppressed. When the frequency of CW interference is the sum of half of the C/A code rate and integral times of PRN code rate, the code tracking error reaches the worst value. The effects of multipath on EMLP increases as the time delay of the reflected signal increases, and then it fluctuates with a value until the time delay is larger than the sum of half of the correlator spacing and the chipping duration. When the time delay is larger than the sum of half of the correlator spacing and the chipping duration, the effect of multipath is suppressed by the code tracking loop.

\section{Acknowledgements}

This work was supported by the National High Technology Research and Development Program of China (863 Program). The fund number is 2011AA120503.

\section{REFERENCES}

[1] P. Misra and P. Enge, “Global Positioning System, Sig- 
nals, Measurements and Performance,” Second Edition, Ganga Jumuna Press, Lincoln, 2006.

[2] E. D. Kaplan and C. J. Hegarty, "Understanding GPS: Principles and Applications,” 2th Edition, Artech House Inc., Norwood, 2006.

[3] M. Irsigler, J. A. Avila-Rodriguez and G. Hein, "Criteria for GNSS Multipath Performance Assessment," Proceedings of the 18th International Technical Meeting of the Satellite Division of the Institute of Navigation, Long Beach, 14-16 September 2005, pp. 2166-2177.

[4] J. W. Betz, "Effect of Narrowband Interference on GPS Code Tracking Accuracy," Proceedings of the $2000 \mathrm{Na}$ tional Technical Meeting of The Institute of Navigation, Anaheim, 26-28 January 2000, pp. 16-27.

[5] A. T. Balaei, A. G. Dempster and L. L. Presti, "Characterization of the Effects of CW and Pulse CW Interference on the GPS Signal Quality" IEEE Transactions on Aerospace and Electronic Systems, Vol. 45, No. 4, 2009, pp. 1418-1431. doi:10.1109/TAES.2009.5310308

[6] J. W. Betz, "Effect of Partial-Band Interference on Receiver Estimation of C/N0: Theory," Proceedings of the 2001 National Technical Meeting of The Institute of $\mathrm{Na}$ - vigation, Long Beach, 22-24 January 2001, pp. 817-828.

[7] J. Jang, M. Paonni and B. Eissfeller, "CW Interference Effects on Tracking Performance of GNSS Receivers," IEEE Transactions on Aerospace and Electronic Systems, Vol. 48, No. 1, 2012, pp. 243-258. doi:10.1109/TAES.2012.6129633

[8] Y. Liu, Y. Ran, T. Ke and X. Hu, "Code Tracking Performance Analysis of GNSS Signal in the Presence of CW Interference," Signal Processing, Vol. 91, No. 4, 2011, pp. 970-987. doi:10.1016/j.sigpro.2010.09.022

[9] B. Motella, S. Savasta, D. Margaria and F. Dovis, "Method for Assessing the Interference Impact on GNSS Receivers," IEEE Transactions on Aerospace and Electronic Systems, Vol. 47, No. 2, 2011, pp. 1416-1431. doi:10.1109/TAES.2011.5751267

[10] Y. Yang, R. R. Hatch and R. T. Sharpe, “GPS Multipath Mitigation in Measurement Domain and Its Applications for High Accuracy Navigation," Proceedings of the 17th International Technical Meeting of the Satellite Division of The Institute of Navigation, Long Beach, 21-24 September 2004, pp. 1124-1130. 